\title{
Consensus Development of Priority Outcome Domains for Community Mental Health Cares by Multiple Stakeholders: Protocol for an Online Delphi Study in Japan
}

\section{Takuma Shiozawa ( $\nabla$ t.shiozawa@ncnp.go.jp )}

NCNP: Kokuritsu Seishin Shinkei Center https://orcid.org/0000-0002-9307-576X

\section{Sosei Yamaguchi}

Kokuritsu Seishin Shinkei Center

\section{Makoto Ogawa}

Kokuritsu Seishin Shinkei Center

Makiko Abe

Kokuritsu Seishin Shinkei Center

Takayuki Kawaguchi

Kokuritsu Seishin Shinkei Center

Momoka Igarashi

Kokuritsu Seishin Shinkei Center

Naonori Yasuma

Kokuritsu Seishin Shinkei Center

Chiyo Fujii

Kokuritsu Seishin Shinkei Center

\section{Study protocol}

Keywords: Delphi method, Community mental health, Outcome domain, Consensus, Patient and Public Involvement, Protocol

Posted Date: July 7th, 2021

DOl: https://doi.org/10.21203/rs.3.rs-669682/v1

License: (c) (1) This work is licensed under a Creative Commons Attribution 4.0 International License. Read Full License 
Version of Record: A version of this preprint was published at Neuropsychopharmacology Reports on October 12th, 2021. See the published version at https://doi.org/10.1002/npr2.12211. 


\section{Abstract}

\section{Background}

The number of patients with mental illness living in the community has been increasing in Japan.

Treatment goals for mental illness have expanded from hospital discharge and improved functioning to employment, living alone, and personal realization. These changes in treatment goals have also influenced mental health research. Recent studies have addressed the development of core outcome sets focusing on clinical aspects of mental illness such as depression and anxiety. However, a well-developed framework of essential outcomes for people with mental illness (service users) who live in the community is lacking. In addition, recent worldwide trends suggest more patient and public involvement (PPI) and the importance of considering multiple stakeholders' views in the area of mental health research. Purpose of this study to explore consensus on high-priority outcome domains among multiple stakeholders such as service users, caregivers, service providers, governmental staff, and researchers in community mental health care and relevant fields in Japan.

\section{Methods}

A three-step approach to developing an outcome list will be used. First, we will develop a long list of outcomes relevant to stakeholders through a literature review of outcomes reported in existing systematic reviews and randomized controlled trials, focus group interviews with key stakeholders, and two online questionnaire surveys of service users and caregivers. Second, the long list will be checked and revised in a two rounds of pilot study. Third, the long list will be shortened to the outcome list through the Delphi methodology with participation from multiple stakeholders. Three rounds of Delphi survey will be conducted and consensus reached if at least $70 \%$ of the participants considered at 'very important' or 'important'. Outcome items that were indeterminate will be taken to the next round. All responses of survey will be feedback to participants in the next round.

\section{Discussion}

Identifying important common outcome domains through collaboration among service users, caregivers, and other stakeholders appears to contribute to the development of evidence for community mental health research in Japan. In addition, the study process itself may help promote PPI in education, practice, and research in the field of community mental health.

\section{Background}

Proper outcome selection is essential for the research and political contexts in the community mental health care setting. Given the recent spread of evidence-based policy making, reasonable outcome selection for multiple stakeholders in each culture has become increasingly important. 
In Japan, the number of service users who live in the community has gradually increased through the deinstitutionalization of patients with mental illness. Accordingly, the treatment goal for mental illness has been shifting to hospital discharge and improved functioning as well as independent living and internal self-realization [1]. Changes in treatment goals have influenced mental health research [1]. For example, in addition to clinical outcomes (e.g., duration of admission, readmissions, symptoms, functioning, etc.), there has been growing interest in measuring outcomes related to personal recovery [2]. As the outcomes of interest increase and diversify, researchers have been seeking a consensus on outcome selection. This is occurring for the community mental health setting in Japan.

Some international projects on outcome consensus had been launched. For example, the Core Outcome Measures in Effectiveness Trials (COMET) Initiative was established to address the issues of inconsistent outcome selection and bias in outcome reporting. The Core Outcome Set (COS) has been developed in each clinical area [3]. The COMET Initiative identified the need for core outcome sets across a wide range of health disciplines and their role in coordinating this information [4]. In addition, the International Consortium for Health Outcomes Measurement (ICHOM) was established in 2012 to redefine the value of health care through patient-centered outcomes [5]. It has started to develop a standard set of outcomes, placing importance on the participation of patients themselves. In summary, the process of outcome selection has received increased attention. In the field of psychiatry, previous studies have developed core outcome sets that should be assessed in research studies. For example, COMET and ICHOM have developed core outcome sets focusing on clinical aspects of mental illness such as depression, anxiety [6], and bipolar disorder [7].

While a core set of psychiatric outcomes has been developed, there are some considerations when developing priority outcome domains for community mental health care. First, community mental health service providers generally serve people with severe mental illness who have a variety of diagnoses and do not always offer diagnosis-specific services. Community mental health now assumes that have a significant capacity to manage their own illnesses and to pursue personally goals, even people with the most severe mental illnesses or ongoing symptoms [8]. Current mental health trends and interventions thus emphasize self-management, self-agency, strengths, resilience, and capacity for functional recovery [9]. In other words, community mental health service users have similar needs, goals, and interests regardless of their diagnosis. Second, community mental health services are usually comprehensive and provided by a multidisciplinary team rather than through a single intervention method. For example, while a staff member in an assertive services term provides employment services, another member provides cognitive behavioral therapy. Therefore, priority outcome lists that are diagnosis-specific or only focus only on a specific intervention type may not be applicable to community mental health care. In addition, this study will be conducted only with stakeholders in Japan. The reason for restricting this study to participants living in Japan is that the priority outcome domains in the field of community mental health care may differ from culture to culture because of differences in the nature of disability welfare services and the perception of mental illness in each country. 
Insufficient involvement of multiple stakeholders is another concern for outcome selection in community mental health care. The outcomes that researchers and patients have rated as important have not always been the same $[10,11]$. In addition, the involvement of only service users is inadequate for selecting reasonable outcomes. A proper outcome selection process for community mental health care requires involvement from diverse stakeholders such as caregivers, service providers, and government staff [12]. The lack of involvement and an outcome selection framework may lead to irrelevant outcome measures, which may be ultimately a barrier for accumulating appropriate evidence about community mental health services $[13,14]$. Indeed, a recent worldwide trend suggests that the concept of patient and public involvement (PPI) and incorporation of the views of service users and other stakeholders is important in mental health research [12]. In addition, PPI is an useful approach not only for intervention studies but also for outcome selection research [15].

The above limitations based on recent trends and features of community mental health care suggest that the issue of outcome selection or priority outcome domain. However, few studies have addressed culturespecific consensus building for outcome selection, comprehensive outcome selection that does not focus on a particular diagnosis or intervention, or multiple stakeholder involvement in community mental health services. To address this evidence gap, we will conduct a Delphi survey with multiple stakeholders such as service users, caregivers, service providers, national and regional government staff, and researchers in Japan. The participation of diverse stakeholders will enable the identification of important common and diverse outcomes for community mental health care in Japan.

\section{OBJECTIVES}

The purpose of this study is to explore consensus on important and high-priority outcome domains for multiple stakeholders in community mental health care and relevant research in Japan.

\section{Methods}

\section{Overview}

A three-step approach to identify the priority outcome domains will be used. First, a draft comprehensive list of outcomes will be compiled through a review of outcomes reported in Cochrane reviews and existing randomized controlled trials, focus group interviews with key stakeholders, and online surveys of service users and caregivers. Second, the comprehensive outcome list will be refined through a pilot study. Third, a Delphi study will be conducted using the comprehensive outcome list to develop outcome lists (Fig. 1). Our research team includes researchers with backgrounds in academic medicine, nursing, social work, occupational therapy, music therapy, clinical psychology, and service user experience. The multidisciplinary research team has conducted or will conduct all the stages of this study and report the findings. In other words, all the process of the study has involved or will involve user-researchers.

The authors assert that all procedures contributing to this work comply with the ethical standards of the relevant national and institutional committees on human experimentation and with the Helsinki 
Declaration of 1975, as revised in 2008. This study protocol was approved by the Tokyo Metropolitan University Ethics Committee (No. 20083) and the Research Ethics Committee of the National Center of Neurology and Psychiatry (No. A2021-005). The study protocol was registered in the University Hospital Medical Information Network (UMIN) Clinical Trials Registry (UMIN-CTR ID, UMIN000044680).

\section{Step 1: Development of a comprehensive list of outcomes that are considered important by service users, caregivers, and stakeholders in Japanese community mental health research}

To inform the design of the Delphi surveys, the sections on Steps 1 and 2 describe what we have already done. However, no substantive findings are presented in this paper. During Step 1, we developed a long list of outcomes using the results from focus group interviews and online questionnaires, in addition to the data collected through a literature review.

\section{1-1. Literature review}

A review of literature was conducted to ensure identification of an exhaustive list of relevant outcome domains. We took this approach with the goal of comprehensively examining the outcomes that researchers have used in past studies. Details of the literature review can be found in an article by Igarashi et al (Igarashi et al, in submission). A literature search was performed using electronic databases (Cochrane Library, MEDLINE, PsycINFO, and Ichushi-Web) for any original research published in English or Japanese. We searched for articles using a combination of keyword searches related to community mental health care and severe mental illness such as "severe/serious mental illness" and "community mental health" and all articles for the leading to the search date were reviewed. Papers were therefore excluded first on the basis of their titles and then on the basis of their abstracts. Details of the inclusion and exclusion criteria are shown in Supplementary Table 1. Title and abstract screening and full-text screening were independently completed by at least two authors for Cochrane Library (MI, TK, and SY), MEDLINE, PsycINFO (TK and SY), and Ichushi-Web (TS and SY). Any discrepancies were resolved through discussion with a co-author (CF). For each article, we extracted all outcomes specified in the Methods section. More specifically, we identified outcomes that indicated what factor was measured rather than descriptions of how it was measured. We classified the outcomes identified from each article and added them to the long list of outcomes.

\section{1-2. Focus group interviews}

In September 2019, group interviews were conducted with service users and other stakeholders. The first half of the group interviews were conducted with the same stakeholders and the second half of the interviews were conducted with a mixture of stakeholders. Interviews were conducted to examine outcomes that stakeholders, including service users and caregivers, thought were important. The details of this research are described in an article by Ogawa et al (Ogawa et al, in preparation).

\section{Participants and recruitment}

Service users, caregivers, community mental health service professionals (e.g., doctors, nurses, psychiatric social workers, occupational therapists, clinical psychologists), national or regional government staff that work in mental health, and researchers who have studied community mental health 
participated in the group interviews. Inclusion criteria were (1) ability to respond to the interview content voluntarily, (2) living in Japan, and (3) native Japanese speaker. Exclusion criteria were (1) current hospitalization, (2) age under 20 years, and (3) adult guardianship. We recruited participants by explaining the purpose and content of this study to service users or caregiver organizations, professional associations, and academic societies. Regarding participants with a research background, we recruited authors of original papers on community psychiatry and community mental health services in addition to recruiting members of relevant academic societies. The following participants joined the group interviews: 10 persons with mental illness, 6 caregivers, 10 community mental health service workers, 4 government staff, and 11 community mental health researchers. We explained the purpose of the study and the details of the research methodology to the participants. Written informed consent was obtained from all participants.

\section{Methods}

In the group interviews, participants were divided into groups according to each attribute and discussed the theme of "What are the outcomes that should be measured in research on community psychiatry and community mental health services?" One research team member participated in each group as a facilitator to encourage and organize input. At the end of the group interviews, the outcome results from each group were shared with all groups. The content of the interviews were audio recorded and transcribed. In addition, basic information on the participants such as age and gender collected before the interview was also tabulated.

\section{Analysis of the focus group interviews}

We transcribed the recorded interviews. We collected statements that we judged to be about "outcomes that should be measured in research on community psychiatry and community mental health services." The validity of the text for analysis was ensured by having the research team members (SY, MO, NY, and MA) work in pairs to confirm the text and adopt the parts that one or more of pair judged to be relevant for analysis. We summarized the extracted statements and coded them as outcome items. The coded outcome items were categorized and combined with similar items by research team members (MO, NY, and TS).

\section{1-3. Online questionnaire surveys}

\section{Study design}

In order to collect a wide range of potential outcomes from the perspective of service users and caregivers, we conducted two online surveys. One was an online cross-sectional survey of caregivers (Yasuma et al, in submission). The other survey was an online survey of service users who lived in the community (Shiozawa et al, in preparation).

\section{Participants and recruitment}
(1) Caregivers 
Participants were recruited from the LINE Family Association of Schizophrenia. This family association had 238 registered members as of August 1, 2020. It offered a platform for information exchange and peer support for caregivers of people with schizophrenia using the LINE online software. Eligibility criteria were as follows: (a) caregiver of a person with schizophrenia and (b) age over 20 years. For example, a caregiver can be someone who cares for a family member with schizophrenia and looks after his or her daily living needs.

\section{(2) Service users}

Participants were recruited from the Community Mental Health \& Welfare Bonding Organization (COMHBO). The COMHBO had 500 registered members who are "Combo Writer" service users that introduce their experiences in an institutional journal. Eligibility criteria were as follows: (a) ability to respond to the interview content voluntarily and (b) age over 20 years. Exclusion criteria were (a) current hospitalization and (b) adult guardianship.

\section{Methods}

The explanatory text of this survey and the URL of the website for the questionnaire survey were sent to potential participants via the mailing list of their organizations. Potential participants were asked to access the website. Potential participants read the details of the explanatory text about the study at the beginning of the web page and continued to answer only if they agreed to participate in the study. In addition to the basic information of participants, we set an open-ended question to capture the important outcomes for service users or caregivers of people with schizophrenia in community mental health research. The question was worded as: "What do you want to become in the future after receiving support? Please enter up to three of your own ideas."

\section{Analysis of the online questionnaire surveys}

Three authors (NY, TK, and TS) independently extracted the participants' responses for outcomes. They adjusted for word fluctuations in the responses. Next, similar responses were collected and categorized through joint analysis and thorough discussion by research team members (NY, TK, and TS). During the categorization process, outcomes with ultimately unclear meanings were excluded.

\section{1-4. Development of the long list of outcomes}

The long list of outcome domains was generated by combining the results from the literature review, focus group interviews, and online questionnaire surveys. We extracted 853 outcome items from the literature review, 81 outcome items from the focus group interviews, and 91 items from the two online surveys.

To form an accessible Delphi survey, the outcomes needed to be grouped together where there were areas of commonality or duplication and mapped into outcome domains. Four research meetings were held to remove duplication of outcome items, further consolidate common outcome items, and map outcome domains. Participants in these meetings were from a range of health and social care research backgrounds (doctors, nurses, psychiatric social workers, occupational therapists, clinical psychologists, 
experts-by-experience). In addition, research team members (MI and TS) held multiple meetings to discuss and organize outcome items. All outcome items collected were maintained on an Excel spreadsheet. Research team members laid out the outcome items printed on paper on a table and summarized similar outcome items or deleted outcome items that were determined to be inconsistent with the purpose of this study. Any disagreements were resolved through discussion. Finally, 94 outcome items were identified (e.g., Psychiatric symptoms or mental state; Physical health; Activity or leisure; Employment, life satisfaction, quality of life, or well-being) and included in the Delphi study (Table 1). We defined each outcome item and created a summary to understand the meaning of the items. 
Table 1

Long list of outcome domains

\section{Death - suicide}

2. Death - all causes

3. Psychiatric symptoms or mental state

4. Psychological distress (anxiety or depression)

5. Relapse or remission

6. Insight

7. Substance use

8. Self-harm

9. Violence or aggression

10. Suicidal ideation or attempt

11. Laboratory measures

12. Physical health

13. Weight and obesity

14. Physical fitness

15. Chronic pain

16. Self-care

17. Perceived stress

18. Subjective health status

19. Overall functioning

20. Daily living skills

21. Contact with the legal system

22. Interpersonal relations

23. Help-seeking

24. Partner or marriage

25. Overall social functioning

26. Independent living

27. Social connectedness

\section{Religion or beliefs}

42. Sexual satisfaction

43. Cognitive functioning

44. Knowledge of illness and services

45. Medication adherence

46. Treatment adherence

47. Attitudes towards medication or treatment

48. Satisfaction with services

49. Unmet needs

50. Perceived coercion

51. Housing stability

52. Earnings

53. Duration of community living

54. Family relationships or functioning

55. Living with family

56. Having a role model

57. Therapeutic relationship

58. Costs of mental health care

59. Costs of all care

60. Mental health service use

61. Hospital admission

62. Involuntary treatment

63. Duration of admission

64. Outpatient visits

65. Emergency service use

66. Non-psychiatric service use

67. Number of caregivers needed to maintain stable state 


\section{Death - suicide}

28. Activities or leisure

29. Place of safety and belonging

30. Employment

31. Work-related skills or vocational ability

32. Absence from or leaving a job

33. Job hunting and related activities

34. Duration of employment or hours worked

35. Childbirth and childcare

36. Caregiving for family members

37. Education

38. Role in society

39. Peer support

40. Life satisfaction, quality of life, or wellbeing

81. Motivation

82. Empowerment or self-determination

83. Self-esteem

84. Resilience

85. Feeling dependent on psychiatric treatments

86. Symptom control skills or coping

87. Stigma and discrimination

\section{Religion or beliefs}

68. Medication prescription

69. Adverse events or effects

70. Caregivers' mental health

71. Caregivers' physical health

72. Caregivers' subjective health status

73. Family's stigma and discrimination

74. Caregivers' problem-solving or coping skills

75. Caregivers' self-esteem

76. Caregivers' knowledge of illness and services

77. Caregivers' service satisfaction

78. Caregivers' life satisfaction or quality of life

79. Expressed emotions

80. Caregivers' service use

88. Social support for caregivers

89. Experience of caregiving

90. Burden of care

91. Financial burden of care

92. Influence on caregivers' lifestyles

93. Influence on caregivers' jobs

94. Influence on caregivers' leisure activities

\section{Step 2: Pilot study of the outcome list}

We will conduct a pilot study for the purpose of determining the adequacy of the long list and descriptions for each outcome item developed in Step 1. The pilot study also aims to obtain feedback on wording, usability, and visuality for the online survey form.

\section{Participants and recruitment}


Participants in the pilot study will be service users, caregivers, community mental health service providers, government staff, and community mental health researchers. We will recruit two participants from each group.

\section{Data collection and analysis}

In the pilot study, two rounds of online questionnaire surveys will be conducted using a website.

$<$ Round one $>$

(1) We will send the participants an invitation to the survey website by e-mail. We will ask them to respond to the survey from the dedicated webpage. Potential participants will read the explanation of the study at the beginning of the webpage and then will answer only if they agree to participate in the study.

(2) The long list of outcomes generated during the Step 1 will be presented to participants. Participants will rate each outcome item in the long list on a seven-point scale from "very important" to "not important at all." In addition, participants can write comments if they have opinions on each outcome item. They can also suggest new outcome items. Furthermore, the usability of the survey website and the clarity of the descriptions will be evaluated.

(3) We will compile and summarize the participants' comments. Results will be presented as averages, percentage of respondents with each score, and variance so that participants can confirm the variation in others' opinions. In order to avoid the risk of adjustment bias by our research team, all comments will be reflected in the summary. Corrections to wording and additions of items to the long item list will be made after discussion among research team members.

$<$ Round two $>$

(1) We will report the results of the first round to the participants. After confirming the results of the first round, participants will respond to the revised long list of outcomes.

(2) Participants will rate each outcome in the revised long list on a seven-point scale from "very important" to "not important at all." Participants will enter and submit free-text descriptions for each outcome.

Using the results of the pilot survey responses, we will draft the "Long list of outcomes for community mental health research in Japan" to be used in the Delphi survey.

\section{Step 3: Delphi study}

An online Delphi survey will be conducted to develop the priority outcome list for the community mental health setting in Japan. Since there are no definitive or optimal methods for conducting a Delphi survey, we will determine the survey methods accordingly with reference to previous studies.

\section{1) Number of study participants}


The Delphi survey for five panel attributes will include service users, caregivers, community mental health service providers, national or regional government staff that work in mental health, and community mental health researchers. While there is no clear criterion for the number of participants in a Delphi study, studies usually include 50 members per panel, according to a relevant review [16]. With reference to previous studies, the number of expected participants in this study will be 250 , consisting of 50 people for each panel attribute.

\section{2) Response rate}

In general, a response rate over $70 \%$ is required to obtain reliable results in Delphi studies [17]. This study will follow previous studies and aim for a response rate of $70 \%$ or higher for each round.

\section{3) Number of iterations for consensus building}

Given the risk of participant burden and thus a lower response rate, a Delphi study in the field of health services generally needs two to three rounds for consensus building $[18,19]$. Based on previous studies, this study will include a total of three rounds.

\section{4). Criteria for consensus}

The definition of consensus in a prior study was $70 \%$ or more of respondents rating an outcome item as "important" or "very important" [17]. In a review article, the basic definition was set in the range of 55$100 \%$, and since $70 \%$ was reported the most of using as criterion [20]. The definition of consensus for this study is $70 \%$ or more. In addition, we will exclude items if $75 \%$ or more of the respondents rated them as "not important" or "not important at all" based on a past core outcome study of discharge interventions among service users [21].

\section{Participants and recruitment}

The potential participants of the Delphi survey will be stakeholders in community mental health care, as in the pilot surveys. There will be 50 stakeholders of each type. Considering a 11.7\% attrition rate (about 6 participants) based on a previous study [22], we will recruit approximately 60 participants in each stakeholder group. Inclusion criteria include (1) ability to respond to the study voluntarily and (2) living in Japan. Exclusion criteria include (1) currently hospitalization, (2) age under 20 years, and (3) adult guardianship.

Other inclusion criteria will be established for each attribute panel in this study. The inclusion criteria for service users are: (1) diagnosis of mental illness (potential participants with dementia and intellectual disability are excluded from participation), (2) disability that affects daily life (serious mental illness), and (3) use of community mental health and social services. The inclusion criteria for caregivers are: (1) primary caregiver for a service user. The inclusion criteria for community mental health service providers are: (1) national license in medical health and welfare that have (doctor, nurse, psychiatric social worker, occupational therapist, clinical psychologist, and other) and (2) history of involvement in community mental health and welfare services. This study will recruit at least five people from each major mental health profession (doctor, nurse, psychiatric social worker, occupational therapist, clinical psychologist) in order to reduce the occupational bias of participating supporters. The inclusion criteria for national or 
regional government staff are: (1) working for a government agency and (2) involvement in work related to community mental health and social services. The inclusion criteria for researchers are: (1) doing research in the field of community mental health care. We also asked whether the researcher held a national license in medical health and welfare. As in the community mental health service providers, we will recruit at least five people from each mental health profession (doctor, nurse, psychiatric social worker, occupational therapist, clinical psychologist, and other) to participate in the study.

We will recruit participants by explaining the purpose and content of this study to services user or caregiver organizations, professional associations, and academic societies. We will explain the survey outline to key persons in each organization. If the organizations or key persons agree to collaborate on the Delphi survey, they will inform their fellow members about this survey. We will also ask the participate to focus group interview participants and authors of the original papers on community psychiatry and community mental health services.

\section{Delphi Round 1}

In the first round, participants will be asked to register online. Each participant will be asked to identify the applicable stakeholder group. A unique identifier will be assigned to each participant to allow identification of individuals that complete each round. Potential participants who are informed about the Delphi study via announcements from associations or emails from the research team will visit a webpage introducing the Delphi study web page of Department of Community Mental Health \& Law (URL:https://www.ncnp.go.jp/nimh/chiiki/research/32.html). If an individual is interested in participating in the Delphi study, they will provide an email address and obtain an invitation URL for the survey website. As in the pilot study, participants will read the detailed explanation of the study at the beginning of the webpage. They indicate agreement to study participation by participating in the survey. The long list identified through Steps 1 and 2 will be presented to participants. Participants will rate each outcome item on a seven-point scale from "very important" to "not important at all." In addition, participants can submit comments for each outcome item. They can also suggest new outcome items if they think that there are essential outcome items that were not in the long outcome list.

\section{Round 1 data analysis}

The response rate will be assessed at the end of Round 1. The total number of respondents completing the round will be compared to the number of respondents who agreed to participate in this study. Response rate will be calculated by the number of participants overall and by stakeholder group (service user, caregiver, community mental health service providers, government staff, and researcher). For each outcome item, the distribution of scores will be summarized and analyzed. For each outcome item in Round 1, the proportion of participants scoring 1-2,3-5, and 6-7 on the seven-point Likert scale will be calculated for each item. In addition, the results will be presented using averages, percentages of respondents with each score, and variance so that variations in opinions can be confirmed. Each outcome will be classified as "consensus in," "consensus out," or "no consensus." "Consensus in," referring to consensus that the outcome should be included in a core outcome set, will be defined as greater than $70 \%$ of participants scoring $6-7$ and less than $25 \%$ of participants scoring $1-2$. "Consensus 
out," referring to consensus that the outcome item is not appropriate for include in the priority outcome domain, will be defined as greater than $75 \%$ of participants scoring $1-2$ and less than $50 \%$ of participants scoring 6-7 in all stakeholder groups. "No consensus" is defined as any other distribution of scores. Only outcomes identified as "no consensus" will be re-evaluated in Round 2.

All free-text comments will be reflected in the summary to avoid the risk of adjustment bias introduced by the research team. Modifications to the wording of items or addition of outcomes based on free-text comments will be made after discussion among the research team members.

\section{Delphi Round 2}

We will report the results of Round 1 to the participants. After confirming the Round 1 results, participants will respond to the revised questionnaire. Participants will rate each outcome item on a seven-point scale from "very important" to "not important at all." In addition, if participants have any comments on each outcome item, they could write them in the free-text field.

\section{Round 2 data analysis}

The response rate will be assessed at the end of Round 2 using the same procedure as in Round 1. In Round 2, changes in the number of respondents from Round 1 will also be assessed. For each outcome item, the number of respondents and distribution of scores will be summarized and analyzed. For each outcome in Round 2, the proportion of participants scoring 1-2, 3-5, and 6-7 on the seven-point Likert scale will be calculated. In addition, the results will be presented using averages, percentages of respondents with each score, and variance so that the participants can understand the various views of others. Based on the consensus criteria, only those outcomes that are identified as "no consensus" will be re-evaluated in Round 3.

\section{Delphi Round 3}

We will report the results of Round 2 to the participants. The participants will confirm the Round 2 results. Next, they will respond to the revised questionnaire. As with Round 2, participants will rate each outcome items on a seven-point scale to indicate their priority regarding the outcome items in the list.

\section{Round 3 data analysis}

At the end of Round 3, the response rate and number of respondents will be assessed using the same procedure as in the previous rounds. The number of respondents and distribution of scores will be summarized and analyzed for each outcome item. Round 3 data will be analyzed by stakeholder group. Results on outcome items will be presented using averages, percentages of respondents with each score, and variance. Each outcome will be classified as "consensus in," "consensus out," or "no consensus."

Based on the results of Rounds 1-3 of the Delphi survey, the outcomes categorized as "consensus in" by all stakeholder groups will be on the "Important outcome domain list for service users, caregivers, supporters, and researchers in community mental health research in Japan."

\section{Discussion}


Clarifying what are important outcomes is an essential process for developing effective services and policies. This study aims to develop consensus on key outcome domains for community mental health care among service users, caregivers, service providers, national and regional government staff, and researchers in Japan. The priority outcome domains identified in this study might contribute to proper outcome selection in future research. This study will identify the outcome areas that each stakeholder group considers to be high priority outcome domains for community mental health in Japan. This helps increase the understanding of common or disparate interests of research outcome domains between stakeholders. While international trends have emphasized the importance of PPI in the research context, few studies have addressed this issue in Japan. We involved a user-researcher in all stages of the study process. For example, we adopted an idea from a user-researcher for develop a web system that participants will be can temporarily store their answers in the middle of a research. This system allows participants to take a break and participate in the survey. It might reduce the burden of the survey. In addition, the pilot study follows the process of modifying the wording from the preliminary survey so that participants from any attribute panel can easily understand the survey process and the contents of the Delphi survey. The collaborative process of this study itself might contribute to promotion of PPI in a Japanese community mental health service setting.

This study protocol has several strengths. First, the long outcome list will be developed using multiple strategies, including a literature review, focus group interviews, and web-based surveys. This process enables the creation of a comprehensive list of outcomes for community mental health care. Second, the protocol of the Delphi survey clearly shows the criteria for consensus-building. While there are various approaches to conducting a Delphi survey, this protocol paper has established the criteria as much as possible by referring to previous studies.

This study has some potential limitations. First, the use of an online survey method for the Delphi study restricts participation to individuals who have access to a computer and the internet. Thus, our study does not reflect the views of those who cannot use access the internet or a computer for any reason. Second, this study will only identify the outcome items with relatively high interest. In other words, this study will present the outcome domains considered to be important by key stakeholders, but it will not suggest particular scales or measurement methods for specific outcomes. For example, if the participants consider psychiatric symptoms as an important outcome, we will not mention any particular scale (e.g., Positive And Negative Syndrome Scale or Brief Psychiatric Rating Scale).

Despite some limitations, this study has several benefits. The development of the outcome domain list through collaboration with multiple stakeholders will make the common important outcomes more visible. This domain list is expected to contribute to the development of evidence for community mental health research in Japan in the future. Moreover, this research process highlights the importance of involving service users and caregivers and joint decision-making, not just the interests of researchers. This study process will help promote PPI in the field of community mental health care in Japan.

\section{Declarations}




\section{Ethics approval and consent to participate}

The ethical considerations of the current study, including the informed consent process and patient privacy measures, are based on the ethics guidelines for medical research in Japan. The study protocol was approved by the ethics committees of the Tokyo Metropolitan University (No. 20083) and the National Center of Neurology and Psychiatry, Japan (A2021-005). If we amend our protocol, we will ask the ethics committees to review the amendments and we will report the change in the manuscript.

\section{Consent for publication}

Not applicable.

\section{Availability of data and materials}

The datasets generated and/or analyzed during the current study are not publicly available due to a relevant Japanese policy for Ethical Guidelines for Medicine for a person of interest and the ethical committee approval for this study.

\section{Competing interests}

The authors declare that they have no competing interests.

\section{Funding}

This work is supported by the Research and Development Fund for Mental and Neurological Disorders, "Fundamental Research on Effective Community Life Support Systems for People with Severe Mental Disorders and Their Families."

\section{Authors' contributions}

TS is the principal investigator responsible for the initial draft of this manuscript and organizing and implementing the study. TS, SY, MO, MA, TK, MI, NY, and CF conceived this work. SY and CF secured the funding. All the authors contributed to the development and refinement of the study protocol. All authors read and approved the final manuscript.

\section{Acknowledgements}

Not applicable

\section{References}

1. Thornicroft G, Slade M: New trends in assessing the outcomes of mental health interventions. World Psychiatry 2014, 13(2):118-124. 
2. Slade $M$, Amering M, Oades L: Recovery: an international perspective. Epidemiologia e Psichiatria Sociale 2008, 17(2):128-137.

3. Williamson PR, Altman DG, Blazeby JM, Clarke M, Devane D, Gargon E, Tugwell P: Developing core outcome sets for clinical trials: issues to consider. Trials 2012, 13(1):132.

4. Initiative C: Core Outcome Measures in Effectiveness Trials. 2011.

5. ICHOM.

6. Obbarius A, Van Maasakkers L, Baer L, Clark DM, Crocker AG, De Beurs E, Emmelkamp PMG, Furukawa TA, Hedman-Lagerlöf E, Kangas $M$ et al: Standardization of health outcomes assessment for depression and anxiety: recommendations from the ICHOM Depression and Anxiety Working Group. Quality of Life Research 2017, 26(12):3211-3225.

7. Retzer A, Sayers R, Pinfold V, Gibson J, Keeley T, Taylor G, Plappert H, Gibbons B, Huxley P, Mathers $\mathrm{J}$ et al: Development of a core outcome set for use in community-based bipolar trials-A qualitative study and modified Delphi. PLOS ONE 2020, 15(10):e0240518.

8. Mueser KT, Corrigan PW, Hilton DW, Tanzman B, Schaub A, Gingerich S, Essock SM, Tarrier N, Morey B, Vogel-Scibilia S et al: Illness Management and Recovery: A Review of the Research. Psychiatric Services 2002, 53(10):1272-1284.

9. Graham Thornicroft GS, Kim T. Mueser, Robert E. Drake: Oxford Textbook of Community Mental Health. Oxford university Press 2011:406pp.

10. Mease PJ, Arnold LM, Crofford LJ, Williams DA, Russell IJ, Humphrey L, Abetz L, Martin SA: Identifying the clinical domains of fibromyalgia: Contributions from clinician and patient delphi exercises. Arthritis \& Rheumatism 2008, 59(7):952-960.

11. Chalmers I, Glasziou P: Avoidable waste in the production and reporting of research evidence. The Lancet 2009, 374(9683):86-89.

12. Boardman J: Routine outcome measurement: recovery, quality of life and co-production. The British Journal of Psychiatry 2018, 212(1):4-5.

13. Williamson PR, Gamble C, Altman DG, Hutton JL: Outcome selection bias in meta-analysis. Stat Methods Med Res 2005, 14(5):515-524.

14. Dwan K, Altman DG, Arnaiz JA, Bloom J, Chan A-W, Cronin E, Decullier E, Easterbrook PJ, Von Elm E, Gamble $\mathrm{C}$ et al: Systematic Review of the Empirical Evidence of Study Publication Bias and Outcome Reporting Bias. PLOS ONE 2008, 3(8):e3081.

15. Smith H, Horobin A, Fackrell K, Colley V, Thacker B, Hall DA: Defining and evaluating novel procedures for involving patients in Core Outcome Set research: creating a meaningful long list of candidate outcome domains. Research Involvement and Engagement 2018, 4(1).

16. Gargon E, Crew R, Burnside G, Williamson PR: Higher number of items associated with significantly lower response rates in COS Delphi surveys. Journal of Clinical Epidemiology 2019, 108:110-120.

17. Sumsion T: The Delphi Technique: An Adaptive Research Tool. British Journal of Occupational Therapy 1998, 61(4):153-156. 
18. Green $B$, Jones $M$, Hughes D, Williams A: Applying the Delphi technique in a study of GPs' information requirements. Health Soc Care Community 1999, 7(3):198-205.

19. Thangaratinam S, Redman CW: The Delphi technique. The Obstetrician \& Gynaecologist 2005, 7(2):120-125.

20. Vernon W: The Delphi technique: A review. International Journal of Therapy and Rehabilitation 2013, 16(2).

21. Tyler N, Wright N, Grundy A, Waring J: Developing a core outcome set for interventions to improve discharge from mental health inpatient services: a survey, Delphi and consensus meeting with key stakeholder groups. BMJ Open 2020, 10(5):e034215.

22. Fukasawa M, Suzuki Y, Nakajima S, Asano K, Narisawa T, Kim Y: Systematic Consensus Building on Disaster Mental Health Services After the Great East Japan Earthquake by Phase. Disaster Med Public Health Prep 2015, 9(4):359-366.

\section{Figures}




\begin{tabular}{|l|}
\hline Step 1 \\
Development of \\
a comprehensive \\
list of outcomes
\end{tabular}

Step 2

Pilot study

\section{Step 3}

Delphi study
1-1. Literature review May 2020 to October 2020

853 items identified

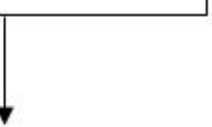

1-2. Focus group interviews

September 2019

81 items identified
1-3. Online questionnaire surveys

August 2020 and

January 2021

91 items identified

Comprehensive list of outcomes that are considered important by service users, caregivers, and stakeholders in Japanese community mental health research

June 2021

Delphi pilot study (Rounds 1 and 2)

2 service users, 2 caregivers, 2 community mental health care providers, 2 national or regional government staff, and 2 researchers specializing in community mental health care will participate
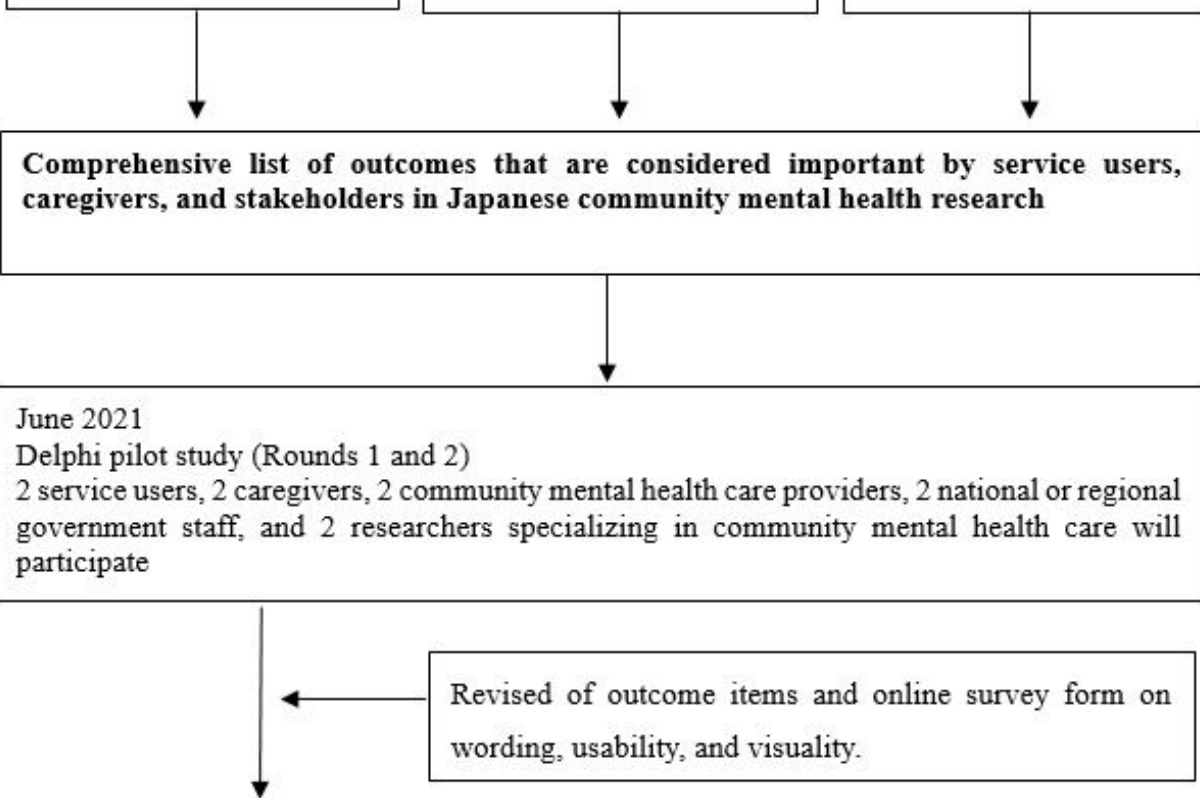

Draft of "Long list of outcomes to be measured in community mental health research in Japan"

July 2021 to October 2021

Delphi study (Round 3)

50 service users, 50 caregivers, 50 community mental health care providers, 50 national or regional government staff, and 50 researchers specializing in community mental health care will participate

\section{Figure 1}

\section{Supplementary Files}

This is a list of supplementary files associated with this preprint. Click to download. 
- SupplementaryTable1.docx

Page 21/21 